\title{
Bootstrap Confidence Intervals of the Difference between Two Process Capability Indices for Half Logistic Distribution
}

\author{
Wararit Panichkitkosolkul \\ Department of Mathematics and Statistics \\ Faculty of Science and Technology \\ Thammasat University, Phathum Thani, Thailand \\ wararit@mathstat.sci.tu.ac.th \\ Somchit Wattanachayakul \\ Department of Mathematics and Statistics \\ Faculty of Science and Technology \\ Thammasat University, Phathum Thani, Thailand \\ somjit@mathstat.sci.tu.ac.th
}

\begin{abstract}
The process capability indices are important numerical measures in statistical quality control. Well-known process capability indices are constructed under the process distribution is normal. Unfortunately, this situation is rather not realistic. This paper focuses on the half logistic distribution. The bootstrap confidence intervals for the difference between two process capability indices for the mentioned distribution are proposed. The bootstrap confidence intervals considered in this paper consist of the standard bootstrap confidence interval, the percentile bootstrap confidence interval and the bias-corrected percentile bootstrap confidence interval. A Monte Carlo simulation has been used to investigate the estimated coverage probabilities and average widths of the bootstrap confidence intervals. Simulation results showed that the estimated coverage probabilities of the percentile bootstrap confidence interval and the bias-corrected percentile bootstrap confidence interval get closer to the nominal confidence level than those of the standard bootstrap confidence interval.
\end{abstract}

Keywords: Process capability index, Bootstrap confidence interval, Half logistic distribution.

\section{Introduction}

The half logistic distribution, which is the distribution of the absolute logistic random variable, was introduced by Balakrishnan (1985). The main references about the half logistic distribution include Balakrishnan and Chan (1992), Balakrishnan and Wong (1994) and Balakrishnan and Aggarwala (1996). If $Y$ is a logistic random variable, then $X=|Y|$ has a half logistic distribution. The probability density function $(f(x))$ and the cumulative distribution function $(F(x))$ are

$$
f(x)=\frac{2 \exp \{-(x-\mu) / \sigma\}}{\sigma[1+\exp \{-(x-\mu) / \sigma\}]^{2}},
$$

and

$$
F(x)=\frac{1-\exp \{-(x-\mu) / \sigma\}}{[1+\exp \{-(x-\mu) / \sigma\}]}, \quad x \geq \mu \geq 0, \quad \sigma>0,
$$

where $\mu$ and $\sigma$ are the location and the scale parameters, respectively. Characterizations of the half logistic distribution were described in Olapade and Ojo (2002). The graph of 
the probability density function for half logistic distribution is shown in Fig. 1.1 The mean and the variance of $X$ are defined as

$$
E(X)=\mu+\sigma \ln (4) \text { and } \operatorname{Var}(X)=\sigma^{2}\left[\frac{\pi^{2}}{3}-(\ln (4))^{2}\right]
$$

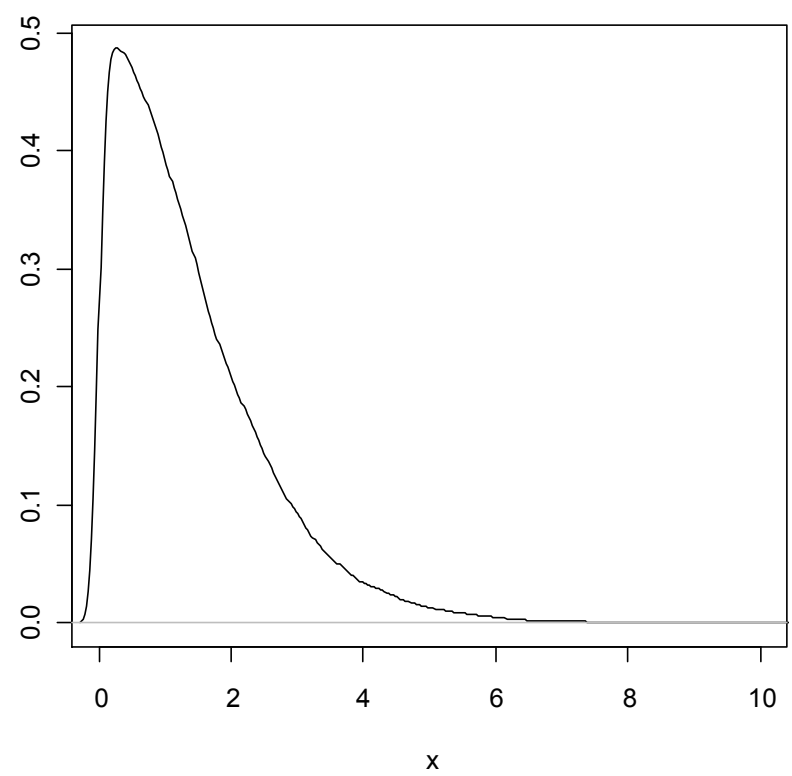

Figure 1.1 The probability density function for half logistic distribution with $\mu=0$ and $\sigma=1$.

Several studies have applied the half logistic distribution. For instance, Balakrishnan (1985) has suggested the usage of this distribution as a possible life-time model with an increasing hazard rate. In addition, Balakrishnan and Chan (1992) have shown that the failure times of air conditioning equipment in a Boeing 720 airplane fits the half logistic distribution quite well. This distribution was also applied to environmental and sports records data (Mbah and Tsokos, 2008). In recent papers, many authors have applied the half logistic distribution under progressive Type-II censoring (see Kang et al., 2008, Balakrishnan and Saleh, 2011, Jang et al. 2011). As mentioned above, it is known that the half logistic distribution is an increasing failure rates model with reasonable importance in statistical quality control and reliability studies (see Kantam and Rosaiah 1998,Kantam et al. 2000, Srinivasa, 2004, Satyaprasad, 2007, Rosaiah et al., 2009, Kantam et al., 2010).

One of the statistical quality control tools widely used is the process capability index (PCI). This index uses both process variability and process specification to determine whether the process is capable (Peng, 2010). Even though there are many process capability indices, the two most commonly used indices are $C_{p}$ and $C_{p k}$ (Kane, 1986, Zhang, 2010). In this paper, we focus only on the popular process capability index $C_{p k}$ defined as follows

$$
C_{p k}=\min \left\{\frac{U S L-\mu}{3 \sigma}, \frac{\mu-L S L}{3 \sigma}\right\},
$$


where USL and LSL denote respectively the upper and lower specification limits of the process, $\mu$ is the process mean and $\sigma$ is the process standard deviation. As the process standard deviation and the process mean are unknown, they must be estimated from the sample data $\left\{X_{1}, \ldots, X_{n}\right\}$. Under the normality assumption, the sample mean $\bar{X}$; $\bar{X}=n^{-1} \sum_{i=1}^{n} X_{i}$ and the sample standard deviation $S ; S=\sqrt{(n-1)^{-1} \sum_{i=1}^{n}\left(X_{i}-\bar{X}\right)^{2}}$ are used to estimate the unknown parameters $\mu$ and $\sigma$ in Eq. (1.3), respectively. Therefore, the natural estimator of the process capability index $C_{p k}$ can be obtained as

$$
\tilde{C}_{p k}=\min \left\{\frac{U S L-\bar{X}}{3 S}, \frac{\bar{X}-L S L}{3 S}\right\} \text {. }
$$

However, the underlying process distribution is non-normal in some situations. Hence, it may be a skewed distribution. To deal with these phenomena, Clements (1989) proposed a new method for computing the estimator of the process capability index $C_{p k}$ when the process distribution is non-normal. This estimator is defined as

$$
\breve{C}_{p k}=\min \left\{\frac{U S L-M}{U_{p}-M}, \frac{M-L S L}{M-L_{p}}\right\},
$$

where $U_{p}, L_{p}$ and $M$ denote the $99.865^{\text {th }}$ percentile, the $0.135^{\text {th }}$ percentile and the $50^{\text {th }}$ percentile of the distribution, respectively. The advantage of $\breve{C}_{p k}$ shown in Eq.(1.4) is that it can be applied to any distribution. Kantam et al. (2010) discussed the relationship between $\breve{C}_{p k}$ and the probability that a product falling outside the specification limits when $X$ has a half logistic distribution. This probability is given by

$$
P_{t}=P(X \leq L S L)+P(X \geq U S L)=1+F(L S L)-F(U S L),
$$

where $F(\cdot)$ is the cumulative distribution function of a half logistic distribution shown in Eq.(1.2). Using the open source statistical package R (Ihaka and Gentleman 1996), some values of $L_{p}, U_{p}$ and $M$ for the half logistic distribution are shown in Table 1.

Table 1: The values of $L_{p}, U_{p}$ and $M$ for the half logistic distribution

\begin{tabular}{|c|c|c|c|c|}
\hline$\mu$ & $\sigma$ & $L_{p}$ & $U_{p}$ & $M$ \\
\hline 0 & 1 & 0.002700002 & 7.300123 & 1.098612 \\
0 & 1.5 & 0.00405 & 10.95018 & 1.647918 \\
\hline 0.5 & 1 & 0.5027 & 7.800123 & 1.598612 \\
0.5 & 1.5 & 0.50405 & 11.45018 & 2.147918 \\
\hline 1 & 1 & 1.0027 & 8.300123 & 2.098612 \\
1 & 1.5 & 1.00405 & 11.95018 & 2.647918 \\
\hline 1.5 & 1 & 1.5027 & 8.800123 & 2.611111 \\
1.5 & 1.5 & 1.50405 & 12.45018 & 3.147918 \\
\hline 2 & 1 & 2.0027 & 9.300123 & 3.098612 \\
2 & 1.5 & 2.00405 & 12.95018 & 3.647918 \\
\hline
\end{tabular}


The estimator $\breve{C}_{p k}$ in Eq. (1.4) can be applied when the scale parameter $\sigma$ of the half logistic distribution is equal to 1 . Therefore, if a scale parameter $\sigma$ is introduced and known, i.e., $\sigma \neq 1$ in Eq.(1.1), the optimal estimator of $C_{p k}$ is given by

$$
\breve{C}_{p k}^{\prime}=\min \left\{\frac{U S L-\sigma M}{\sigma\left(U_{p}-M\right)}, \frac{\sigma M-L S L}{\sigma\left(M-L_{p}\right)}\right\} .
$$

In practice, the scale parameter $\sigma$ is unknown. Therefore, we must estimate the unknown $\sigma$ by its estimator. In this paper, we use the moment method for calculating this estimator. The estimator of $C_{p k}$ for a half logistic distribution is

$$
\hat{C}_{p k}=\min \left\{\frac{U S L-\hat{\sigma} M}{\hat{\sigma}\left(U_{p}-M\right)}, \frac{\hat{\sigma} M-L S L}{\hat{\sigma}\left(M-L_{p}\right)}\right\},
$$

where $\hat{\sigma}$ is the estimator of $\sigma$. Here, we use the simple estimator which is computed by the moment method given by $\hat{\sigma}=\frac{1}{\ln (4)}[\bar{X}-\hat{\mu}]$ and the maximum likelihood estimator for $\mu$ is $\hat{\mu}=X_{(1)}$, the smallest sample order statistics. The moments of the half logistic distribution were shown in Giles (2012).

In this paper, our focus is on the difference between two process capability indices, $C_{p k 1}-C_{p k 2}$, which we will denote by $\delta$ which

$$
\delta=\min \left\{\frac{U S L-\mu_{1}}{3 \sigma_{1}}, \frac{\mu_{1}-L S L}{3 \sigma_{1}}\right\}-\min \left\{\frac{U S L-\mu_{2}}{3 \sigma_{2}}, \frac{\mu_{2}-L S L}{3 \sigma_{2}}\right\},
$$

where $\mu_{1}, \mu_{2}$ and $\sigma_{1}, \sigma_{2}$ are the process mean and standard deviation of the first and the second population, respectively. Similar to Eq.(1.5), we can get the estimator of $\delta$ which is

$\hat{\delta}=\min \left\{\frac{U S L-\hat{\sigma}_{1} M_{1}}{\hat{\sigma}_{1}\left(U_{p 1}-M_{1}\right)}, \frac{\hat{\sigma}_{1} M_{1}-L S L}{\hat{\sigma}_{1}\left(M_{1}-L_{p 1}\right)}\right\}-\min \left\{\frac{U S L-\hat{\sigma}_{2} M_{2}}{\hat{\sigma}_{2}\left(U_{p 2}-M_{2}\right)}, \frac{\hat{\sigma}_{2} M_{2}-L S L}{\hat{\sigma}_{2}\left(M_{2}-L_{p 2}\right)}\right\}$,

where $\hat{\sigma}_{1}$ and $\hat{\sigma}_{2}$ are the moment estimator of $\sigma_{1}$ and $\sigma_{2}$ given by $\hat{\sigma}_{1}=\frac{1}{\ln (4)}\left[\bar{X}_{1}-\hat{\mu}_{1}\right]$ and $\hat{\sigma}_{2}=\frac{1}{\ln (4)}\left[\bar{X}_{2}-\hat{\mu}_{2}\right]$, respectively, $\hat{\mu}_{1}=X_{1(1)}$ and $\hat{\mu}_{2}=X_{2(1)}$.

The remainder of the paper is organized as follows. Section 2 describes the bootstrap confidence intervals. Some simulation evidence on the performance of bootstrap confidence intervals is provided in Section 3. In Section 4, all bootstrap confidence intervals are illustrated and compared through numerical example. A discussion of the 
results and conclusions are presented in the final section. The conclusions are offered in the final section.

\section{Bootstrap Confidence Intervals}

The bootstrap is a computer-based and resampling method for assigning measures of accuracy to statistical estimates (Efron and Tibshirani, 1993). Many types of bootstrap methods for constructing confidence intervals have been introduced; for example, the standard bootstrap method (SB), the percentile bootstrap method (PB) and the biascorrected percentile bootstrap method (BCPB).

For a sequence of independent and identically distributed (i.i.d.) random variables, the bootstrap procedure can be defined as follows (Tosasukul et al., 2009). Let the random variables $\left\{X_{k, j}^{*}, 1 \leq j \leq m\right\}$ be the result from sampling $m$ times from the $k^{\text {th }}$ population with replacement from the $n$ observations $X_{k, 1}, \ldots, X_{k, n}$. The random variables $\left\{X_{k, j}^{*}, 1 \leq j \leq m\right\}$ are called the bootstrap samples from original data $X_{k, 1}, \ldots, X_{k, n}$. In what follows, we describe the constructions for the confidence interval of the difference between two process capability indices $\delta$ using bootstrap techniques.

\subsection{Standard Bootstrap (SB) Confidence Interval}

Let $X_{k, b}^{*}$, where $1 \leq b \leq B$, and $k=1,2$ be the $b^{\text {th }}$ bootstrap samples and let $X_{k, 1}^{*}, \ldots, X_{k, B}^{*}$ be the $B$ bootstrap samples. The $b^{\text {th }}$ bootstrap estimator of $\delta$ is computed by

$$
\begin{aligned}
& \hat{\delta}^{*(b)}=\min \left\{\frac{U S L-\hat{\sigma}_{1}^{*(b)} M_{1}}{\hat{\sigma}_{1}^{*}\left(U_{p 1}-M_{1}\right)}, \frac{\hat{\sigma}_{1}^{*(b)} M_{1}-L S L}{\hat{\sigma}_{1}^{*(b)}\left(M_{1}-L_{p 1}\right)}\right\}-\min \left\{\frac{U S L-\hat{\sigma}_{2}^{*(b)} M_{2}}{\hat{\sigma}_{2}^{*(b)}\left(U_{p 2}-M_{2}\right)}, \frac{\hat{\sigma}_{2}^{*(b)} M_{2}-L S L}{\hat{\sigma}_{2}^{*(b)}\left(M_{2}-L_{p 2}\right)}\right\}, \\
& \text { where } \hat{\sigma}_{1}^{*(b)}=\bar{X}_{1}^{*(b)} / \ln (4), \bar{X}_{1}^{*(b)}=n_{1}^{-1} \sum_{j=1}^{n_{1}} X_{1, j}^{*}, \hat{\sigma}_{2}^{*(b)}=\bar{X}_{2}^{*(b)} / \ln (4), \bar{X}_{2}^{*(b)}=n_{2}^{-1} \sum_{j=1}^{n_{2}} X_{2, j}^{*}, \text { Thus, }
\end{aligned}
$$

the standard bootstrap $(1-\alpha) 100 \%$ confidence interval is

$$
C I_{S B}=\left(\overline{\hat{\delta}}^{*}-Z_{1-\frac{\alpha}{2}} S_{\delta}^{*}, \overline{\hat{\delta}}^{*}+Z_{1-\frac{\alpha}{2}} S_{\delta}^{*}\right)
$$

where $Z_{1-\alpha / 2}$ is a $(1-\alpha / 2)^{t h}$ quantile of the standard normal distribution, $\overline{\hat{\delta}}^{*}=B^{-1} \sum_{i=1}^{B} \hat{\delta}^{*(i)}$ and

$$
S_{\delta}^{*}=\sqrt{\frac{1}{B-1} \sum_{i=1}^{B}\left(\hat{\delta}^{*(i)}-\overline{\hat{\delta}}^{*(i)}\right)^{2}}
$$




\subsection{Percentile Bootstrap (PB) Confidence Interval}

The percentile bootstrap $(1-\alpha) 100 \%$ confidence interval is given by

$$
C I_{P B}=\left(\hat{\delta}_{\left(\frac{\alpha}{2} B\right)}^{*}, \hat{\delta}_{\left(\left[1-\frac{\alpha}{2}\right] B\right)}^{*}\right)
$$

where $\hat{\delta}_{(r)}^{*}$ is the $r^{\text {th }}$ ordered value on the list of the $B$ bootstrap estimator of $\delta$.

\subsection{Bias-Corrected Percentile Bootstrap (BCPB) Confidence Interval}

The obtained bootstrap distributions using only a sample of the complete bootstrap distribution may be shifted higher or lower than would be expected. Therefore, this approach has been introduced in order to correct for the potential bias. Firstly, using the ordered distribution of $\hat{\delta}^{*}$, compute the probability $P_{0}=P\left(\hat{\delta}^{*} \leq \hat{\delta}\right)$. Then, $Z_{0}=\Phi^{-1}\left(P_{0}\right)$. Therefore, the percentile of the ordered distribution $G^{*}\left(\hat{\delta}^{*}\right), P_{L}=\Phi\left(2 Z_{0}-Z_{1-\alpha / 2}\right)$ and $P_{U}=\Phi\left(2 Z_{0}+Z_{1-\alpha / 2}\right)$ are obtained, where $\Phi(\cdot)$ is the standard normal cumulative function. Finally, the bias-corrected percentile bootstrap $(1-\alpha) 100 \%$ confidence interval is defined as follows

$$
C I_{B C P B}=\left(\hat{\delta}_{\left(P_{L} B\right)}^{*}, \hat{\delta}_{\left(P_{U} B\right)}^{*}\right),
$$

where $\hat{\delta}_{(r)}^{*}$ is the $r^{t h}$ ordered value on the list of the $B$ bootstrap estimator of $\delta$.

To study the different confidence intervals, we consider their estimated coverage probabilities and average widths. For each of the methods considered, the probability that the true value of $C_{p k}$ is covered by the $(1-\alpha) 100 \%$ bootstrap confidence interval, which is called the "coverage probability", can be obtained. In addition, the average width of the bootstrap confidence interval is calculated based on the $M=5,000$ different trials. The estimated coverage probability and the average width are given by

$$
\widehat{1-\alpha}=\frac{\#(L \leq \delta \leq U)}{M},
$$

and

$$
\widehat{\text { Width }}=\frac{\sum_{i=1}^{M}\left(U_{i}-L_{i}\right)}{M},
$$

where $L$ and $U$ denote the lower and upper bound of the bootstrap confidence interval.

In the following section, a simulation study is presented in order to evaluate the performance of the confidence intervals $C I_{S B}, C I_{P B}$, and $C I_{B C P B}$ based on their estimated coverage probabilities and average widths. 


\section{Simulation Study}

A simulation study on the behavior of three bootstrap confidence intervals of the difference between two process capability indices for half logistic distribution is described. The statistical package $\mathrm{R}$ (Ihaka and Gentleman, 1996) is used to carry out the simulation study in this section. In addition, the sample sizes and parameter values of half logistic distribution that we used in this simulation are listed in Table 2. Similar to previous experiments of Kantam et al. (2010), we set the lower and upper specification limits are 1 and 29, respectively. The process capability indices and the difference between the two process capability indices of twelve designs are shown in Table 2. For each design, $B=1,000$ bootstrap samples with each of size $n$ are drawn from the original sample. Additionally, the simulation is replicated 5,000 times. The $90 \%$ and $95 \%$ bootstrap confidence intervals are constructed by each of the three methods, i.e., SB, PB, and BCPB confidence intervals.

The simulation results are summarized in Tables 3 and 4 . These two tables present the results on the estimated coverage probabilities and average widths of $90 \%$ and $95 \%$ bootstrap confidence intervals, respectively. We begin with the results for Designs 1a-f, 2a-f, 3a-f and 4a-f $\left(\mu_{1}<\sigma_{1}\right.$ and $\left.\mu_{2}<\sigma_{2}\right)$, the estimated coverage probabilities of $C I_{S B}$ are larger than the nominal confidence level. In addition, the estimated coverage probabilities of $C I_{B C P B}$ get reasonably closer to the nominal confidence level than those of $C I_{S B}$ and $C I_{P B}$ for all sample sizes. When Designs 6a-f and 7a-f $\left(\mu_{1}=\sigma_{1}\right.$ and $\left.\mu_{2}<\sigma_{2}\right)$ are considered, the estimated coverage probabilities of $C I_{S B}$ are not less than the nominal confidence level. The $C I_{P B}$ provides the estimated coverage probabilities closer to the nominal confidence level than those of $C I_{S B}$ and $C I_{B C P B}$.

As one can see, under Designs 5a-f, 8a-f, 9a-f, 10a-f, 11a-f and 12a-f $\left(\mu_{1}>\sigma_{1}\right.$ and $\left.\mu_{2} \leq \sigma_{2}\right)$, the $C I_{S B}, C I_{P B}$, and $C I_{B C P B}$ give poor coverage probabilities than the nominal confidence level for large sample sizes. On the other hand, the estimated coverage probabilities of $C I_{S B}$ are significantly above the nominal confidence level for small sample size $\left(n_{1}=n_{2}=10\right)$.

The $C I_{B C P B}$ provides the shortest average width for all situations. Additionally, the average widths of all the bootstrap confidence intervals get shorter when $n_{1}$ and (or) $n_{2}$ increases. 
Wararit Panichkitkosolkul, Somchit Wattanachayakul

Table 2: Sample sizes and parameter values of half logistic distribution used in the simulation study.

\begin{tabular}{|c|c|c|c|c|c|c|c|c|c|}
\hline Design & $n_{1}$ & $n_{2}$ & $\mu_{1}$ & $\sigma_{1}$ & $\mu_{2}$ & $\sigma_{2}$ & $C_{p k 1}$ & $C_{p k 2}$ & $\delta$ \\
\hline $\begin{array}{l}1 \mathrm{a} \\
1 \mathrm{~b} \\
1 \mathrm{c} \\
1 \mathrm{~d} \\
1 \mathrm{e} \\
1 \mathrm{f}\end{array}$ & $\begin{array}{l}10 \\
30 \\
50 \\
10 \\
10 \\
30\end{array}$ & $\begin{array}{l}10 \\
30 \\
50 \\
30 \\
50 \\
50\end{array}$ & 0.5 & 1 & 0 & 1 & 0.5462 & 0.0900 & 0.4562 \\
\hline $\begin{array}{l}2 \mathrm{a} \\
2 \mathrm{~b} \\
2 \mathrm{c} \\
2 \mathrm{~d} \\
2 \mathrm{e} \\
2 \mathrm{f}\end{array}$ & $\begin{array}{l}10 \\
30 \\
50 \\
10 \\
10 \\
30\end{array}$ & $\begin{array}{l}10 \\
30 \\
50 \\
30 \\
50 \\
50\end{array}$ & 0.5 & 1.5 & 0 & 1.5 & 0.9011 & 0.5969 & 0.3042 \\
\hline $\begin{array}{l}3 \mathrm{a} \\
3 \mathrm{~b} \\
3 \mathrm{c} \\
3 \mathrm{~d} \\
3 \mathrm{e} \\
3 \mathrm{f}\end{array}$ & $\begin{array}{l}10 \\
30 \\
50 \\
10 \\
10 \\
30 \\
\end{array}$ & $\begin{array}{l}10 \\
30 \\
50 \\
30 \\
50 \\
50\end{array}$ & 0.5 & 1.5 & 0 & 1 & 0.9011 & 0.0900 & 0.8111 \\
\hline $\begin{array}{l}4 \mathrm{a} \\
4 \mathrm{~b} \\
4 \mathrm{c} \\
4 \mathrm{~d} \\
4 \mathrm{e} \\
4 \mathrm{f}\end{array}$ & $\begin{array}{l}10 \\
30 \\
50 \\
10 \\
10 \\
30\end{array}$ & $\begin{array}{l}10 \\
30 \\
50 \\
30 \\
50 \\
50\end{array}$ & 0.5 & 1 & 0 & 1.5 & 0.5462 & 0.5969 & -0.0507 \\
\hline $\begin{array}{l}5 \mathrm{a} \\
5 \mathrm{~b} \\
5 \mathrm{c} \\
5 \mathrm{~d} \\
5 \mathrm{e} \\
5 \mathrm{f}\end{array}$ & $\begin{array}{l}10 \\
30 \\
50 \\
10 \\
10 \\
30 \\
\end{array}$ & $\begin{array}{l}10 \\
30 \\
50 \\
30 \\
50 \\
50 \\
\end{array}$ & 1.5 & 1 & 0.5 & 1 & 1.4535 & 0.5462 & 0.9073 \\
\hline $\begin{array}{l}6 \mathrm{a} \\
6 \mathrm{~b} \\
6 \mathrm{c} \\
6 \mathrm{~d} \\
6 \mathrm{e} \\
6 \mathrm{f}\end{array}$ & $\begin{array}{l}10 \\
30 \\
50 \\
10 \\
10 \\
30\end{array}$ & $\begin{array}{l}10 \\
30 \\
50 \\
30 \\
50 \\
50\end{array}$ & 1.5 & 1.5 & 0.5 & 1.5 & 1.5094 & 0.9011 & 0.6083 \\
\hline
\end{tabular}


Table 2: $\quad$ (Continued)

\begin{tabular}{|c|c|c|c|c|c|c|c|c|c|}
\hline Design & $n_{1}$ & $n_{2}$ & $\mu_{1}$ & $\sigma_{1}$ & $\mu_{2}$ & $\sigma_{2}$ & $C_{p k 1}$ & $C_{p k 2}$ & $\delta$ \\
\hline $7 a$ & 10 & 10 & \multirow[t]{6}{*}{1.5} & \multirow[t]{6}{*}{1.5} & \multirow[t]{6}{*}{0.5} & \multirow[t]{6}{*}{1} & \multirow[t]{6}{*}{1.5094} & \multirow[t]{6}{*}{0.5462} & \multirow[t]{6}{*}{0.9632} \\
\hline $7 b$ & 30 & 30 & & & & & & & \\
\hline $7 \mathrm{c}$ & 50 & 50 & & & & & & & \\
\hline $7 d$ & 10 & 30 & & & & & & & \\
\hline $7 e$ & 10 & 50 & & & & & & & \\
\hline $7 \mathrm{f}$ & 30 & 50 & & & & & & & \\
\hline $8 \mathrm{a}$ & 10 & 10 & \multirow[t]{6}{*}{1.5} & \multirow[t]{6}{*}{1} & \multirow[t]{6}{*}{0.5} & \multirow[t]{6}{*}{1.5} & \multirow{6}{*}{1.4535} & \multirow[t]{6}{*}{0.9011} & \multirow[t]{6}{*}{0.5525} \\
\hline $8 b$ & 30 & 30 & & & & & & & \\
\hline $8 \mathrm{c}$ & 50 & 50 & & & & & & & \\
\hline $8 d$ & 10 & 30 & & & & & & & \\
\hline $8 \mathrm{e}$ & 10 & 50 & & & & & & & \\
\hline $8 \mathrm{f}$ & 30 & 50 & & & & & & & \\
\hline $9 a$ & 10 & 10 & \multirow[t]{6}{*}{2} & \multirow[t]{6}{*}{1} & \multirow[t]{6}{*}{1} & \multirow[t]{6}{*}{1} & \multirow[t]{6}{*}{1.9149} & \multirow[t]{6}{*}{1.0025} & \multirow[t]{6}{*}{0.9125} \\
\hline $9 b$ & 30 & 30 & & & & & & & \\
\hline $9 c$ & 50 & 50 & & & & & & & \\
\hline $9 d$ & 10 & 30 & & & & & & & \\
\hline $9 \mathrm{e}$ & 10 & 50 & & & & & & & \\
\hline $9 f$ & 30 & 50 & & & & & & & \\
\hline $10 \mathrm{a}$ & 10 & 10 & \multirow[t]{6}{*}{2} & \multirow[t]{6}{*}{1.5} & \multirow[t]{6}{*}{1} & \multirow[t]{6}{*}{1.5} & \multirow[t]{6}{*}{1.6862} & 1.2052 & 0.4810 \\
\hline $10 \mathrm{~b}$ & 30 & 30 & & & & & & & \\
\hline $10 \mathrm{c}$ & 50 & 50 & & & & & & & \\
\hline $10 \mathrm{~d}$ & 10 & 30 & & & & & & & \\
\hline $10 \mathrm{e}$ & 10 & 50 & & & & & & & \\
\hline $10 \mathrm{f}$ & 30 & 50 & & & & & & & \\
\hline $11 \mathrm{a}$ & 10 & 10 & 2 & 1.5 & 1 & 1 & 1.6862 & 1.0025 & 0.6837 \\
\hline $11 \mathrm{~b}$ & 30 & 30 & & & & & & & \\
\hline $11 \mathrm{c}$ & 50 & 50 & & & & & & & \\
\hline $11 d$ & 10 & 30 & & & & & & & \\
\hline $11 \mathrm{e}$ & 10 & 50 & & & & & & & \\
\hline $11 \mathrm{f}$ & 30 & 50 & & & & & & & \\
\hline $12 \mathrm{a}$ & 10 & 10 & 2 & 1 & 1 & 1.5 & 0.9149 & 1.2052 & 0.7097 \\
\hline $12 b$ & 30 & 30 & & & & & & & \\
\hline $12 \mathrm{c}$ & 50 & 50 & & & & & & & \\
\hline $12 d$ & 10 & 30 & & & & & & & \\
\hline $12 \mathrm{e}$ & 10 & 50 & & & & & & & \\
\hline $12 \mathrm{f}$ & 30 & 50 & & & & & & & \\
\hline
\end{tabular}


Table 3: The estimated coverage probabilities and average widths of a $90 \%$ bootstrap confidence intervals of the difference between two process capability indices.

\begin{tabular}{|c|c|c|c|c|c|c|}
\hline \multirow{2}{*}{ Design } & \multicolumn{3}{|c|}{ Coverage probabilities } & \multicolumn{3}{|c|}{ Average widths } \\
\hline & SB & PB & $\mathrm{BCPB}$ & SB & PB & $\mathrm{BCPB}$ \\
\hline $1 \mathrm{a}$ & 0.9860 & 0.9142 & 0.9434 & 2.7235 & 2.3111 & 2.3092 \\
\hline $1 b$ & 0.9178 & 0.8908 & 0.8986 & 0.7999 & 0.7885 & 0.7864 \\
\hline $1 \mathrm{c}$ & 0.9052 & 0.8876 & 0.8926 & 0.5622 & 0.5579 & 0.5570 \\
\hline $1 d$ & 0.9698 & 0.8504 & 0.8864 & 1.9125 & 1.6208 & 1.3842 \\
\hline $1 \mathrm{e}$ & 0.9676 & 0.8088 & 0.8644 & 1.8768 & 1.5627 & 1.2446 \\
\hline 1f & 0.9202 & 0.8992 & 0.9000 & 0.6867 & 0.6773 & 0.6691 \\
\hline $2 a$ & 0.9886 & 0.9176 & 0.9356 & 1.2442 & 1.0618 & 1.0666 \\
\hline $2 b$ & 0.9252 & 0.9018 & 0.9092 & 0.3601 & 0.3547 & 0.3543 \\
\hline $2 \mathrm{c}$ & 0.9088 & 0.8942 & 0.8988 & 0.2535 & 0.2515 & 0.2512 \\
\hline $2 d$ & 0.9732 & 0.8386 & 0.8678 & 0.8648 & 0.7358 & 0.6210 \\
\hline $2 \mathrm{e}$ & 0.9708 & 0.8018 & 0.8516 & 0.8467 & 0.7057 & 0.5488 \\
\hline $2 f$ & 0.9186 & 0.9002 & 0.9008 & 0.3089 & 0.3047 & 0.3004 \\
\hline $3 a$ & 0.9784 & 0.8714 & 0.9126 & 2.1340 & 1.7884 & 1.6881 \\
\hline $3 b$ & 0.9138 & 0.8750 & 0.8982 & 0.6330 & 0.6204 & 0.6030 \\
\hline $3 c$ & 0.9016 & 0.8784 & 0.8936 & 0.4437 & 0.4386 & 0.4310 \\
\hline $3 d$ & 0.9624 & 0.8990 & 0.9158 & 1.0235 & 0.9222 & 0.8829 \\
\hline $3 e$ & 0.9622 & 0.8632 & 0.8920 & 0.9557 & 0.8143 & 0.7239 \\
\hline $3 \mathrm{f}$ & 0.9138 & 0.8946 & 0.9002 & 0.4776 & 0.4724 & 0.4713 \\
\hline $4 a$ & 0.9816 & 0.8906 & 0.9242 & 2.0281 & 1.7091 & 1.6198 \\
\hline $4 b$ & 0.9224 & 0.8996 & 0.9042 & 0.6065 & 0.5949 & 0.5792 \\
\hline $4 c$ & 0.9044 & 0.8952 & 0.8894 & 0.4254 & 0.4208 & 0.4141 \\
\hline $4 d$ & 0.9676 & 0.7926 & 0.8490 & 1.8307 & 1.5141 & 1.1599 \\
\hline $4 e$ & 0.9698 & 0.7712 & 0.8378 & 1.8084 & 1.4869 & 1.0934 \\
\hline $4 f$ & 0.9172 & 0.8898 & 0.8932 & 0.5737 & 0.5609 & 0.5311 \\
\hline $5 a$ & 0.9762 & 0.8756 & 0.8960 & 2.3311 & 1.9775 & 1.9818 \\
\hline $5 b$ & 0.7140 & 0.6696 & 0.6810 & 0.6680 & 0.6570 & 0.6535 \\
\hline $5 c$ & 0.5408 & 0.5024 & 0.5144 & 0.4660 & 0.4619 & 0.4603 \\
\hline $5 d$ & 0.9732 & 0.9346 & 0.8624 & 1.4293 & 1.2390 & 1.0725 \\
\hline $5 e$ & 0.9796 & 0.9296 & 0.8416 & 1.3667 & 1.1621 & 0.9149 \\
\hline $5 f$ & 0.7028 & 0.6944 & 0.6408 & 0.5410 & 0.5347 & 0.5318 \\
\hline $6 a$ & 0.9950 & 0.9674 & 0.8834 & 1.1822 & 1.0331 & 1.0809 \\
\hline $6 b$ & 0.9668 & 0.9480 & 0.8308 & 0.4766 & 0.4697 & 0.4637 \\
\hline $6 c$ & 0.9522 & 0.9302 & 0.8152 & 0.3898 & 0.3826 & 0.3727 \\
\hline $6 \mathrm{~d}$ & 0.9800 & 0.8584 & 0.8008 & 0.8248 & 0.7233 & 0.6169 \\
\hline $6 e$ & 0.9780 & 0.8014 & 0.7718 & 0.7997 & 0.6879 & 0.5377 \\
\hline $6 f$ & 0.9580 & 0.9224 & 0.8080 & 0.4366 & 0.4264 & 0.4004 \\
\hline $7 a$ & 0.9844 & 0.9242 & 0.8854 & 2.0087 & 1.7083 & 1.6297 \\
\hline $7 b$ & 0.9392 & 0.9266 & 0.8388 & 0.6882 & 0.6804 & 0.6778 \\
\hline $7 \mathrm{c}$ & 0.9034 & 0.8900 & 0.7992 & 0.5258 & 0.5224 & 0.5210 \\
\hline $7 d$ & 0.9654 & 0.8962 & 0.8520 & 0.9642 & 0.8884 & 0.8558 \\
\hline $7 e$ & 0.9526 & 0.8218 & 0.8088 & 0.8807 & 0.7930 & 0.7105 \\
\hline $7 f$ & 0.9160 & 0.8940 & 0.8084 & 0.5602 & 0.5560 & 0.5532 \\
\hline
\end{tabular}


Table 3: (Continued)

\begin{tabular}{|c|c|c|c|c|c|c|}
\hline \multirow{2}{*}{ Design } & \multicolumn{3}{|c|}{ Coverage probabilities } & \multicolumn{3}{|c|}{ Average widths } \\
\hline & SB & PB & $\mathrm{BCPB}$ & SB & PB & $\mathrm{BCPB}$ \\
\hline $8 a$ & 0.9758 & 0.9174 & 0.8748 & 1.6024 & 1.3555 & 1.3264 \\
\hline $8 b$ & 0.5388 & 0.5572 & 0.4896 & 0.4535 & 0.4457 & 0.4379 \\
\hline $8 c$ & 0.2726 & 0.2882 & 0.2382 & 0.3155 & 0.3125 & 0.3094 \\
\hline $8 d$ & 0.9808 & 0.9274 & 0.8100 & 1.3519 & 1.1249 & 0.8379 \\
\hline $8 \mathrm{e}$ & 0.9854 & 0.9358 & 0.7972 & 1.3362 & 1.1008 & 0.7626 \\
\hline $8 \mathrm{f}$ & 0.5542 & 0.5912 & 0.4688 & 0.4171 & 0.4090 & 0.3888 \\
\hline $9 a$ & 0.9888 & 0.9222 & 0.8988 & 2.0488 & 1.7439 & 1.7968 \\
\hline $9 b$ & 0.8388 & 0.8056 & 0.6588 & 0.6149 & 0.6072 & 0.6120 \\
\hline $9 \mathrm{c}$ & 0.6604 & 0.6472 & 0.4630 & 0.4449 & 0.4410 & 0.4390 \\
\hline $9 d$ & 0.9960 & 0.9616 & 0.8372 & 1.2700 & 1.1016 & 0.9289 \\
\hline $9 \mathrm{e}$ & 0.9942 & 0.9442 & 0.8006 & 1.2314 & 1.0381 & 0.7750 \\
\hline $9 f$ & 0.8540 & 0.8584 & 0.5928 & 0.5128 & 0.5064 & 0.4934 \\
\hline $10 \mathrm{a}$ & 0.9898 & 0.9904 & 0.7504 & 1.2419 & 1.1133 & 1.1157 \\
\hline $10 \mathrm{~b}$ & 0.7200 & 0.7948 & 0.4616 & 0.7284 & 0.7174 & 0.6746 \\
\hline $10 \mathrm{c}$ & 0.4158 & 0.4882 & 0.2512 & 0.6060 & 0.6027 & 0.5677 \\
\hline $10 \mathrm{~d}$ & 0.9524 & 0.9718 & 0.6680 & 0.9600 & 0.8664 & 0.7998 \\
\hline $10 \mathrm{e}$ & 0.9450 & 0.9634 & 0.6406 & 0.9339 & 0.8387 & 0.7491 \\
\hline $10 \mathrm{f}$ & 0.6578 & 0.7382 & 0.4466 & 0.7040 & 0.6928 & 0.6577 \\
\hline $11 \mathrm{a}$ & 0.9804 & 0.9554 & 0.7346 & 1.8674 & 1.6222 & 1.4736 \\
\hline $11 b$ & 0.6454 & 0.6536 & 0.3460 & 0.8340 & 0.8251 & 0.7719 \\
\hline $11 \mathrm{c}$ & 0.3336 & 0.3432 & 0.1622 & 0.6690 & 0.6652 & 0.6296 \\
\hline $11 d$ & 0.8978 & 0.8184 & 0.5940 & 1.0482 & 0.9763 & 0.9339 \\
\hline $11 \mathrm{e}$ & 0.8648 & 0.7000 & 0.5480 & 0.9843 & 0.9032 & 0.8342 \\
\hline $11 \mathrm{f}$ & 0.5406 & 0.5200 & 0.2708 & 0.7608 & 0.7525 & 0.7173 \\
\hline $12 \mathrm{a}$ & 0.9876 & 0.9534 & 0.7706 & 1.4432 & 1.2372 & 1.2690 \\
\hline $12 b$ & 0.3914 & 0.4640 & 0.3372 & 0.4655 & 0.4575 & 0.4430 \\
\hline $12 c$ & 0.1342 & 0.1946 & 0.1726 & 0.3400 & 0.3334 & 0.3137 \\
\hline $12 d$ & 0.9974 & 0.9804 & 0.7068 & 1.1992 & 0.9949 & 0.7418 \\
\hline $12 \mathrm{e}$ & 0.9978 & 0.9736 & 0.6778 & 1.1826 & 0.9680 & 0.6520 \\
\hline $12 \mathrm{f}$ & 0.3990 & 0.5064 & 0.2948 & 0.4276 & 0.4158 & 0.3755 \\
\hline
\end{tabular}


Table 4: The estimated coverage probabilities and average widths of a $95 \%$ bootstrap confidence intervals of the difference between two process capability indices.

\begin{tabular}{|c|c|c|c|c|c|c|}
\hline \multirow{2}{*}{ Design } & \multicolumn{3}{|c|}{ Coverage probabilities } & \multicolumn{3}{|c|}{ Average widths } \\
\hline & SB & PB & $\mathrm{BCPB}$ & SB & PB & $\mathrm{BCPB}$ \\
\hline $1 \mathrm{a}$ & 0.9954 & 0.9570 & 0.9728 & 3.3069 & 3.1170 & 3.1227 \\
\hline $1 b$ & 0.9654 & 0.9462 & 0.9528 & 0.9512 & 0.9606 & 0.9571 \\
\hline $1 \mathrm{c}$ & 0.9528 & 0.9400 & 0.9494 & 0.6699 & 0.6728 & 0.6715 \\
\hline $1 d$ & 0.9908 & 0.9022 & 0.9394 & 2.2775 & 2.1122 & 1.7941 \\
\hline $1 \mathrm{e}$ & 0.9908 & 0.8662 & 0.9146 & 2.1888 & 2.0067 & 1.5792 \\
\hline $1 f$ & 0.9654 & 0.9520 & 0.9558 & 0.8164 & 0.8209 & 0.8103 \\
\hline $2 a$ & 0.9976 & 0.9626 & 0.9732 & 1.4716 & 1.3986 & 1.4073 \\
\hline $2 b$ & 0.9678 & 0.9514 & 0.9552 & 0.4299 & 0.4344 & 0.4330 \\
\hline $2 \mathrm{c}$ & 0.9648 & 0.9568 & 0.9576 & 0.3019 & 0.3033 & 0.3029 \\
\hline $2 d$ & 0.9932 & 0.8930 & 0.9280 & 1.0231 & 0.9455 & 0.7918 \\
\hline $2 \mathrm{e}$ & 0.9916 & 0.8532 & 0.8974 & 1.0166 & 0.9146 & 0.7025 \\
\hline $2 f$ & 0.9630 & 0.9496 & 0.9484 & 0.3695 & 0.3714 & 0.3661 \\
\hline $3 a$ & 0.9942 & 0.9206 & 0.9510 & 2.5140 & 2.3620 & 2.2337 \\
\hline $3 b$ & 0.9640 & 0.9278 & 0.9426 & 0.7512 & 0.7508 & 0.7304 \\
\hline $3 c$ & 0.9554 & 0.9330 & 0.9450 & 0.5298 & 0.5287 & 0.5191 \\
\hline $3 d$ & 0.9886 & 0.9492 & 0.9632 & 1.2211 & 1.1845 & 1.1150 \\
\hline $3 e$ & 0.9898 & 0.9178 & 0.9454 & 1.1204 & 1.0527 & 0.9234 \\
\hline $3 \mathrm{f}$ & 0.9650 & 0.9496 & 0.9514 & 0.5718 & 0.5740 & 0.5720 \\
\hline $4 a$ & 0.9956 & 0.9346 & 0.9636 & 2.4300 & 2.2786 & 2.1623 \\
\hline $4 b$ & 0.9684 & 0.9474 & 0.9564 & 0.7191 & 0.7197 & 0.7015 \\
\hline $4 c$ & 0.9494 & 0.9444 & 0.9458 & 0.5041 & 0.5036 & 0.4954 \\
\hline $4 d$ & 0.9936 & 0.8400 & 0.8986 & 2.2065 & 1.9790 & 1.5015 \\
\hline $4 e$ & 0.9910 & 0.8202 & 0.8826 & 2.1534 & 1.9355 & 1.4052 \\
\hline $4 f$ & 0.9644 & 0.9458 & 0.9468 & 0.6838 & 0.6804 & 0.6448 \\
\hline $5 a$ & 0.9934 & 0.9454 & 0.9552 & 2.7516 & 2.6256 & 2.6406 \\
\hline $5 b$ & 0.8296 & 0.7678 & 0.7804 & 0.7938 & 0.7998 & 0.7941 \\
\hline $5 c$ & 0.6688 & 0.6198 & 0.6314 & 0.5564 & 0.5580 & 0.5559 \\
\hline $5 d$ & 0.9908 & 0.9634 & 0.9294 & 1.7202 & 1.6203 & 1.3767 \\
\hline $5 e$ & 0.9922 & 0.9562 & 0.9172 & 1.6604 & 1.5180 & 1.1776 \\
\hline $5 f$ & 0.8142 & 0.8048 & 0.7668 & 0.6440 & 0.6486 & 0.6442 \\
\hline $6 a$ & 0.9992 & 0.9894 & 0.9458 & 1.3956 & 1.3285 & 1.4170 \\
\hline $6 b$ & 0.9890 & 0.9826 & 0.9100 & 0.5677 & 0.5631 & 0.5496 \\
\hline $6 c$ & 0.9770 & 0.9678 & 0.8984 & 0.4672 & 0.4571 & 0.4406 \\
\hline $6 \mathrm{~d}$ & 0.9962 & 0.9226 & 0.8902 & 0.9935 & 0.9049 & 0.7555 \\
\hline $6 e$ & 0.9946 & 0.8868 & 0.8668 & 0.9711 & 0.8665 & 0.6609 \\
\hline $6 f$ & 0.9822 & 0.9634 & 0.8942 & 0.5201 & 0.5062 & 0.4711 \\
\hline $7 a$ & 0.9966 & 0.9572 & 0.9382 & 2.4163 & 2.2504 & 2.1523 \\
\hline $7 b$ & 0.9710 & 0.9646 & 0.9074 & 0.8234 & 0.8279 & 0.8173 \\
\hline $7 \mathrm{c}$ & 0.9642 & 0.9554 & 0.8904 & 0.6273 & 0.6280 & 0.6234 \\
\hline $7 d$ & 0.9882 & 0.9520 & 0.9104 & 1.1507 & 1.1127 & 1.0578 \\
\hline $7 e$ & 0.9866 & 0.8954 & 0.8880 & 1.0548 & 0.9853 & 0.8695 \\
\hline $7 f$ & 0.9682 & 0.9558 & 0.8952 & 0.6677 & 0.6683 & 0.6609 \\
\hline
\end{tabular}


Table 4L: (Continued)

\begin{tabular}{|c|c|c|c|c|c|c|}
\hline \multirow{2}{*}{ Design } & \multicolumn{3}{|c|}{ Coverage probabilities } & \multicolumn{3}{|c|}{ Average widths } \\
\hline & SB & $\mathrm{PB}$ & $\mathrm{BCPB}$ & SB & PB & $\mathrm{BCPB}$ \\
\hline $8 \mathrm{a}$ & 0.9930 & 0.9688 & 0.9396 & 1.9218 & 1.8175 & 1.7892 \\
\hline $8 b$ & 0.6534 & 0.6918 & 0.6328 & 0.5400 & 0.5434 & 0.5338 \\
\hline $8 \mathrm{c}$ & 0.3640 & 0.4066 & 0.3496 & 0.3752 & 0.3762 & 0.3722 \\
\hline $8 \mathrm{~d}$ & 0.9942 & 0.9638 & 0.8946 & 1.6007 & 1.4503 & 1.0628 \\
\hline $8 \mathrm{e}$ & 0.9972 & 0.9598 & 0.8894 & 1.5901 & 1.4303 & 0.9883 \\
\hline $8 f$ & 0.6582 & 0.7190 & 0.6034 & 0.4964 & 0.4962 & 0.4710 \\
\hline $9 \mathrm{a}$ & 0.9968 & 0.9644 & 0.9570 & 2.4256 & 2.3099 & 2.3948 \\
\hline $9 b$ & 0.9206 & 0.8984 & 0.7922 & 0.7335 & 0.7439 & 0.7455 \\
\hline $9 c$ & 0.8010 & 0.7932 & 0.6028 & 0.5315 & 0.5371 & 0.5302 \\
\hline $9 d$ & 0.9992 & 0.9790 & 0.9142 & 1.5307 & 1.4002 & 1.1714 \\
\hline $9 \mathrm{e}$ & 0.9996 & 0.9684 & 0.8758 & 1.4467 & 1.3200 & 0.9618 \\
\hline $9 f$ & 0.9328 & 0.9392 & 0.7234 & 0.6127 & 0.6184 & 0.5933 \\
\hline $10 \mathrm{a}$ & 0.9972 & 0.9974 & 0.8516 & 1.4609 & 1.3769 & 1.3909 \\
\hline $10 b$ & 0.8066 & 0.8806 & 0.5968 & 0.8681 & 0.8325 & 0.7888 \\
\hline $10 \mathrm{c}$ & 0.5388 & 0.6260 & 0.3794 & 0.7216 & 0.7039 & 0.6671 \\
\hline $10 \mathrm{~d}$ & 0.9828 & 0.9928 & 0.8020 & 1.2153 & 1.0196 & 0.9819 \\
\hline $10 \mathrm{e}$ & 0.9790 & 0.9868 & 0.7968 & 1.1313 & 0.9767 & 0.8953 \\
\hline $10 \mathrm{f}$ & 0.7652 & 0.8430 & 0.5828 & 0.8449 & 0.8013 & 0.7703 \\
\hline $11 \mathrm{a}$ & 0.9950 & 0.9788 & 0.8222 & 2.2604 & 2.0928 & 1.8903 \\
\hline $11 b$ & 0.7542 & 0.7744 & 0.4498 & 0.9973 & 0.9760 & 0.9097 \\
\hline $11 \mathrm{c}$ & 0.4502 & 0.4714 & 0.2392 & 0.7959 & 0.7826 & 0.7412 \\
\hline $11 d$ & 0.9652 & 0.9098 & 0.6998 & 1.2288 & 1.1565 & 1.1258 \\
\hline $11 \mathrm{e}$ & 0.9468 & 0.8250 & 0.6356 & 1.1739 & 1.0682 & 1.0338 \\
\hline $11 \mathrm{f}$ & 0.6846 & 0.6726 & 0.3768 & 0.9061 & 0.8765 & 0.8383 \\
\hline $12 \mathrm{a}$ & 0.9982 & 0.9888 & 0.8848 & 1.7307 & 1.6358 & 1.6927 \\
\hline $12 b$ & 0.5478 & 0.6848 & 0.4284 & 0.5510 & 0.5582 & 0.5325 \\
\hline $12 \mathrm{c}$ & 0.2080 & 0.3270 & 0.2272 & 0.4057 & 0.4078 & 0.3776 \\
\hline $12 d$ & 0.9996 & 0.9892 & 0.8044 & 1.4398 & 1.2943 & 0.9522 \\
\hline $12 \mathrm{e}$ & 0.9994 & 0.9818 & 0.7588 & 1.4080 & 1.2479 & 0.8424 \\
\hline $12 \mathrm{f}$ & 0.5546 & 0.7336 & 0.3822 & 0.5026 & 0.4998 & 0.4411 \\
\hline
\end{tabular}

\section{Illustrative example}

In this section, a simulated example is presented to illustrate the bootstrap confidence intervals of the difference between two process capability indices developed in Section 2. The first and second random samples of sizes $n_{1}=n_{2}=20$ are generated from the half logistic distribution with $\mu_{1}=0.5, \sigma_{1}=1.5$ and $\mu_{2}=0, \sigma_{2}=1$, respectively. In this case, 
we set $L S L=1$ and $U S L=29$, the true difference between two process capability indices, $\delta$, is 0.8111 . The first random sample $\left(x_{1}\right)$ generated is

$\begin{array}{llllllllll}0.02 & 0.05 & 0.32 & 0.44 & 0.64 & 0.64 & 0.67 & 0.87 & 1.05 & 1.05 \\ 1.32 & 1.33 & 1.40 & 2.52 & 2.80 & 3.21 & 3.66 & 4.11 & 4.12 & 5.04 .\end{array}$

The second random sample $\left(x_{2}\right)$ generated is

$\begin{array}{llllllllll}0.04 & 0.14 & 0.19 & 0.20 & 0.23 & 0.44 & 0.75 & 0.81 & 0.88 & 1.07 \\ 1.07 & 1.09 & 1.29 & 1.50 & 1.62 & 1.83 & 1.91 & 3.56 & 5.04 & 5.15 \text {. }\end{array}$

In addition, the density plot of generated samples is displayed in Fig. 4.1. Assuming the half logistic distribution for corresponding random variables $X_{1}$ and $X_{2}$, three bootstrap confidence intervals of the difference between two process capability indices with confidence level 95\% are constructed, and they are shown in the following table.

Table 5: Bootstrap confidence intervals and widths of the difference between two process capability indices

\begin{tabular}{|l|c|c|}
\hline Methods & Confidence intervals & Widths \\
\hline SB & $(0.2441,1.2891)$ & 1.0450 \\
PB & $(0.3025,1.2891)$ & 0.9866 \\
BCPB & $(0.2654,1.3003)$ & 1.0349 \\
\hline
\end{tabular}

As presented in Table 5, the true difference between $C_{p k 1}$ and $C_{p k 2}(\delta=0.8111)$ lies in the proposed bootstrap confidence intervals. Additionally, the width of $C I_{P B}$ is shorter than any other confidence intervals by about $5 \%$.

Density plot of $x 1$

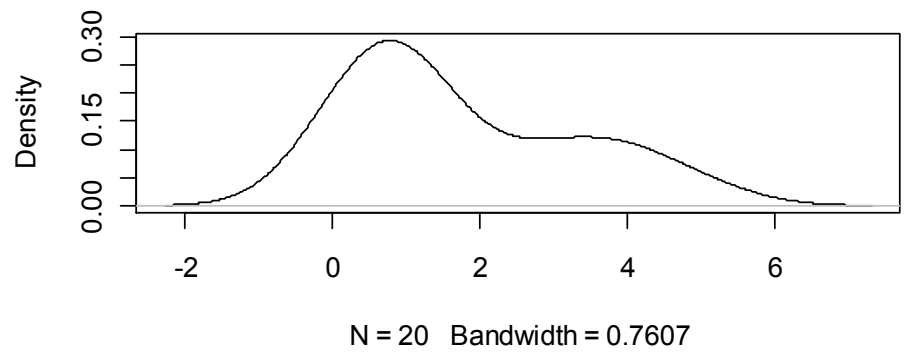

Density plot of $x 2$

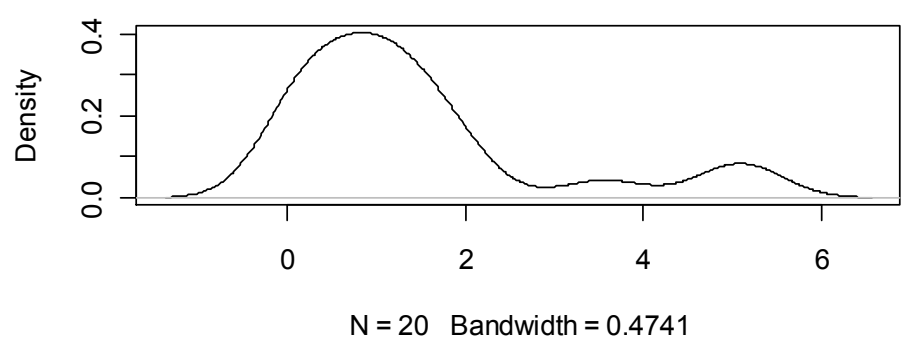

Figure 4.1 The density plot of generated random samples $x_{1}$ and $x_{2}$. 


\section{Conclusions}

In this paper, we have proposed bootstrap confidence intervals of the difference between two process capability indices for half logistic distribution. Three bootstrap confidence intervals are considered: the standard bootstrap confidence interval $\left(C I_{S B}\right)$, the percentile bootstrap confidence interval $\left(C I_{P B}\right)$ and the bias-corrected percentile bootstrap confidence interval $\left(C I_{B C P B}\right)$. The performances of bootstrap confidence intervals are compared by considering their coverage probabilities and average widths using Monte Carlo experiments. Based on simulation studies, the $C_{B C P B}$ achieves better coverage probability than the other bootstrap confidence intervals when $\mu_{1}<\sigma_{1}$ and $\mu_{2}<\sigma_{2}$. In addition, the $C I_{P B}$ performs well with respect to the coverage criterion when $\mu_{1}=\sigma_{1}$ and $\mu_{2}<\sigma_{2}$. On the other hand, proposed bootstrap confidence intervals are not suitable in terms of coverage probability for other situations $\left(\mu_{1}>\sigma_{1}\right.$ and $\left.\mu_{2} \leq \sigma_{2}\right)$.

It would be of interest to propose confidence intervals of the difference between two process capability indices for half logistic distribution when $\mu_{1}>\sigma_{1}$ and $\mu_{2} \leq \sigma_{2}$, and this is left as a topic for future work.

\section{Acknowledgements}

The authors acknowledge the excellent suggestions provided by Professor Dr. R.R.L. Kantam and Professor Dr. David E. Giles. The authors are grateful to Mark Zentz for his careful editing of the manuscript, and to two referees for their constructive comments on an earlier version of this paper.

\section{References}

1. Balakrishnan, N. (1985). Order statistics from the half logistic distribution. Journal of Statistical Computation and Simulation, 20(4): 287-309.

2. Balakrishnan, N. and Aggarwala, R. (1996). Relationships for moments of order statistics from the right-truncated generalized half logistic distribution. Annals of the Institute of Statistical Mathematics, 48(3): 519-534.

3. Balakrishnan, N. and Chan, P.S. (1992). Estimation for the scaled half logistic distribution under Type-II censoring. Computational Statistics \& Data Analysis, 13(2): 123-141.

4. Balakrishnan, N. and Saleh, H.M. (2011). Relations for moments of progressively Type-II censored order statistics from half logistic distribution with applications to inference. Computational Statistics \& Data Analysis, 55(10): 2775-2792.

5. Balakrishnan, N. and Wong K.H.T. (1994). Best linear unbiased estimation of location and scale parameters of the half logistic distribution based on Type-II censored samples. American Journal of Mathematical and Management Sciences, 14(1-2): 53-101.

6. Clements, J.A. 1989. Process capability calculations for non-normal distributions. Quality Progress, 22(9): 95-100. 
7. Efron, B. and Tibshirani, R.J. (1993). An introduction to the bootstrap. Chapman \& Hall, New York.

8. Giles, D.E. (2012). Bias reduction for the maximum likelihood estimators of the parameters in the half-logistic distribution. Communications in Statistics-Theory and Methods, 41(2): 212-222.

9. Ihaka, R. and Gentleman, R. (1996). R: A language for data analysis and graphics. Journal of Computational and Graphical Statistics, 5(3): 299-314.

10. Jang, D.H., Park, J. and Kim, C. (2011). Estimation of the scale parameter of the half-logistic distribution with multiply Type II censored sample. Journal of the Korean Statistical Society, 40(3): 291-301.

11. Kane, V.E. (1986). Process capability indices. Journal of Quality Technology, 18(1): 41-52.

12. Kang, S.B., Cho, Y.S. and Han, J.T. (2008). Estimation for the half logistic distribution under progressive Type-II censoring. Communications of the Korean Mathematical Society, 15(6): 815-823.

13. Kantam, R.R.L. and Rosaiah, K. (1998). Half logistic distribution in acceptance sampling based on life tests. IAPQR Transactions: Journal of the Indian Association for Productivity, Quality \& Reliability, 23(2): 117-125.

14. Kantam, R.R.L., Rosaiah, K. and Anjaneyula, M.S.R. (2000). Estimation of reliability in multicomponent stress-strength model: half logistic distribution. IAPQR Transactions: Journal of the Indian Association for Productivity, Quality \& Reliability, 25(2): 43-52.

15. Kantam, R.R.L., Rosaiah, K. and Subba Rao, R. (2010). Estimation of process capability index for half logistic distribution. International Transactions in Mathematical Sciences and Computer, 3(1): 61-66.

16. Mbah, A.K. and Tsokos, C.P. (2008). Record values from half logistics and inverse weibull probability distribution functions. Neural, Parallel \& Scientific Computations, 16(1): 73-92.

17. Olapade, A.K. and Ojo, M.O. (2002). Characterizations of the Logistic Distribution. Nigerian Journal of Mathematics and Applications, 15(1): 30-36.

18. Peng, C. (2010). Estimating and testing quantile-based process capability indices for processes with skewed distributions. Journal of Data Science, 8(2): 253-268.

19. Rosaiah, K., Kantam, R.R.L. and Srinivasa, R.B. (2009). Reliability test plan for half logistic distribution. Calcutta Statistical Association Bulletin, 61: 241-244.

20. Satyaprasad, R. (2007). Half logistic software reliability growth model, Ph.D. Thesis, Acharya Nagarjuna University, India.

21. Srinivasa, R.B. (2004). Control charts and sampling plans in half logistic model, M. Phil Thesis, Acharya Nagarjuna University, India.

22. Tosasukul, J., Budsaba, K. and Volodin, A. (2009). Dependent bootstrap confidence intervals for a population mean. Thailand Statistician, 7(1): 43-51.

23. Zhang, J. (2010). Conditional confidence intervals of process capability indices following rejection of preliminary tests. Ph.D. Thesis, The University of Texas at Arlington, USA. 